\title{
The Material Point Monte Carlo model: A discrete, off- lattice method for microstructural evolution simulations
}

\author{
Philip E. Goins ${ }^{\mathrm{a}}$ and Elizabeth A. Holm ${ }^{\mathrm{a}, *}$ \\ ${ }^{a}$ Department of Materials Science and Engineering, Carnegie Mellon University, \\ Pittsburgh, PA 15213 USA
}

\begin{abstract}
A new kinetic Monte Carlo model, termed the Material Point Monte Carlo (MPMC) method, is developed to simulate microstructural evolution under a variety of driving forces. The MPMC operates on a spatially random set of discrete material points; at each timestep, points are relocated and their material data are updated according to a statistical mechanics scheme. Because material points are randomly distributed, and remain so under affine deformations, the MPMC eliminates artifacts of the simulation grid that occur in lattice-based methods such as the Monte Carlo Potts model. For model systems the MPMC is verified to correctly reproduce grain boundary motion caused by bulk and interfacial driving forces. It is then applied to larger and more complex problems, including grain growth during plastic deformation.
\end{abstract}

Keywords: Monte Carlo methods; off-lattice methods; mesoscale simulation; grain growth; recrystallization

\footnotetext{
* Corresponding author. Email addresses: pgoins@ andrew.cmu.edu (Philip E. Goins), eaholm@andrew.cmu.edu (Elizabeth A. Holm)
} 


\section{Introduction}

The evolution of microstructure is one of the most important and widely-studied topics in the field of materials science and engineering. A number of modeling techniques have been developed to simulate microstructural evolution in polycrystalline materials, including Monte Carlo Potts model[1-7] multiphase field model[8-11], cellular automata models[12-15], front tracking[16-18], vertex models[19-22], and others[23-25]. Each method possesses strengths and weaknesses. The Monte Carlo Potts model, for instance, is a fast and straightforward method to study grain growth and related phenomena. However, the Potts model suffers from significant lattice artifacts resulting from the symmetry of the grid used[26, 27]. These effects influence not only grain structure and growth, but the behavior of the system under external driving forces. In this paper, we introduce a new kinetic Monte Carlo model, termed the Material Point Monte Carlo (MPMC) model, which does not possess the lattice biases present in the Potts model, and we apply the MPMC method to a number of fundamental microstructural evolution problems.

\section{Methods}

\subsection{Monte Carlo Potts model}

The Monte Carlo Potts model, the starting point for this work, is a $q$-state generalization of the 2-state Ising[28] model initially used to study magnetic domain evolution[1]. The Potts Model was adapted to simulate microstructural evolution in polycrystalline materials [2-4] and has since then become widely used in computational materials science. The simulation begins with an original image mapped onto a $2 \mathrm{D}$ or 3D lattice of $N$ sites; each site is assigned a state corresponding to its grain membership. The system is evolved over time, with $N$ cells being randomly selected and evaluated at each time step. When a cell is selected, a new grain identity state is proposed. The associated energy change from the current state to the new state is computed based on interfacial energy and bulk energy, with the energy of site $i$ given by 


$$
E_{i}=H_{i}+\sum_{i=1 . i \neq}^{n}
$$

where $\eta_{i}$ is the bulk energy of site $i, \gamma_{i j}$ is the interfacial energy of the boundary between sites $i$ and $j$, and $n$ is the number of sites in the neighborhood of site $i$.[29] The probability of accepting the proposed state change generally follows the Metropolis[30] function:

$$
p=\left\{\begin{array}{cc}
M_{i j} & \Delta E \leq 0 \\
M_{i j} e^{-\Delta E / k T} & \Delta E<0
\end{array}\right.
$$

where $\Delta E$ is the energy difference between the initial and proposed state, $k T$ is the simulation temperature, and $M_{i j}$ is the mobility of the interface between sites $i$ and $j$.

This approach evolves the initial system via interfacial and volumetric energy reduction driving forces. Because it is a kinetic Monte Carlo method, time-evolution of the microstructure is also reproduced, and thus relative mobility values are meaningfully captured as well. Overall, the Potts Model can be applied to study a number of microstructural evolution phenomena [31, 32]. However, the Potts model suffers from some unphysical simulation features that arise from its image-based approach. In particular, the regular grid and neighborhood choices give rise to a strong preference for certain grain boundary normal directions in the simulation frame, which can alter grain boundary motion rates in response to driving forces and alter local triple junction angles.[27, 33]

\subsection{Lattice effects in the Potts model}

Several methods have been proposed for reducing or eliminating the lattice artifacts in the Potts model. Alternative grid systems for two-dimensional simulations, such as using a triangular or hexagonal lattices, give the appearance of reduced lattice bias primarily because the natural 120 degree angles in the triangular lattice fortuitously coincide with the equilibrium triple junction angles in isotropic systems [27, 34, 35]. Increasing the simulation temperature is the most common strategy for mitigating lattice effects, but the 
fundamental energetic biases from the lattice remain non-trivial up to the point of boundary disordering [33].

To demonstrate this complication in the Potts model, Figure 1 shows the boundary energy and mobility in an "isotropic" system, computed for flat Potts model boundaries in two and three dimensions. The anisotropic properties that emerge are simulation-space artifacts of the cubic grid. Here, energy is defined as the sum of the energy of the flat boundary normalized by its area:

$$
\gamma(\theta)=\frac{H}{A}=\frac{\sum_{i=1}^{N} \sum_{j=1}^{n} \delta_{i j}}{A}
$$

where $\gamma$ is the interfacial energy of a given boundary inclination, $H$ is the sum of the energy of the boundary in the system, $A$ is the area of the boundary, $N$ is the number of sites in the system, $n$ is the number of points in a neighborhood, and $\delta$ is 0 if the grain identities are alike at site $i$ and $j$, and 1 if there are unlike. An "effective mobility" term is also defined as the instantaneous probability of an orientation state flip along the boundary, using a $k T$ value of 1 , and computed as the total probability of orientation flips at the boundary, divided by the area of the boundary:

$$
M_{f}(\theta)=\frac{\sum_{i=1}^{N} p_{i j}+p_{j i}}{A}
$$

where, $M_{f}$ is the effective mobility of a boundary, indicating the instantaneous rate of movement from fluctuation that can be expected from that plane, $p$ is the probability of flipping from the current grain identity value at a site to the orientation state across the boundary. 


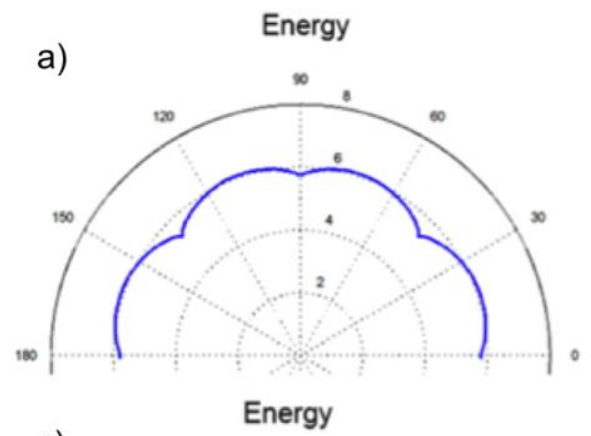

c)

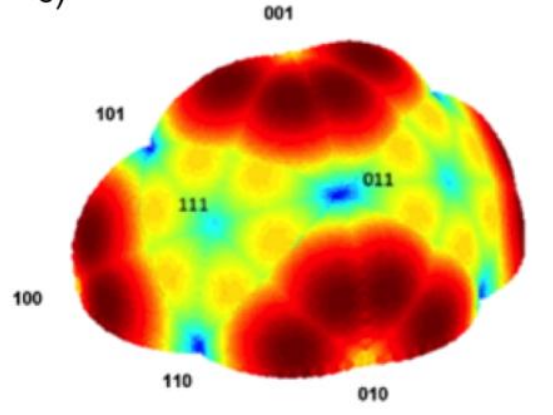

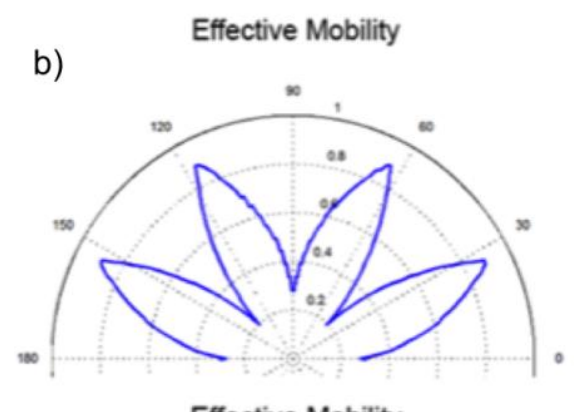

d)

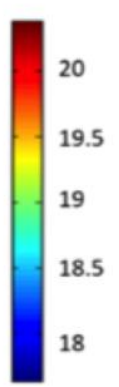

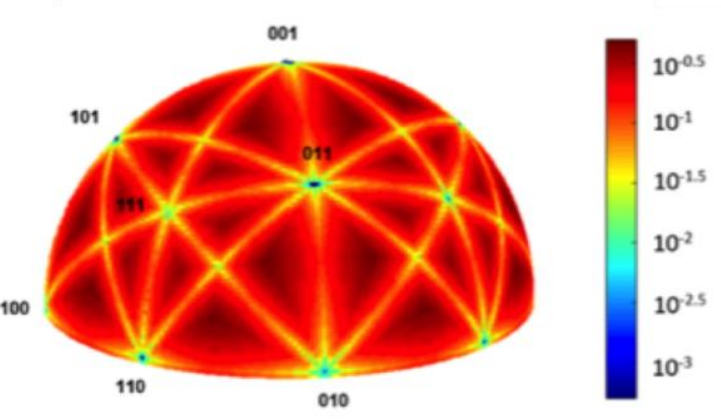

Figure 1: (a) The interfacial energy and (b) effective mobility of a flat, Potts model boundary in the 2D square lattice with first and second neighbor interactions as a function of inclination in the simulation frame. (c) The interfacial energy and (d) effective mobility of a flat, Potts model boundary in the 3D simple cubic lattice with first, second, and third neighbor interactions as a function of grain boundary normal direction in simulation reference frame.

In $2 \mathrm{D}$ the highest energy boundary inclination has an interfacial energy about 1.13 times larger than the lowest energy boundary inclination, as shown in Figure 1(a), which has also been reported by Glazier et. al[36]. However, the instantaneous rate of boundary motion can vary by nearly one order of magnitude, even with $k T$ values of 1 , due to the local configuration of orientation states along the boundary, as shown in Figure 1(b). Thus, the impact of anisotropy in the simulation can be very strong. This effect is actually stronger in $3 \mathrm{D}$, where the interfacial energy can vary by a factor of 1.25 , and the $k T=1$ mobility can vary by nearly three orders of magnitude as a function of boundary inclination in sample space. These thermodynamic and kinetic anisotropies can have significant impact on microstructural morphology and evolution. 
Recently, Mason et al. derived a long-range Gaussian kernel for computing the local Potts model neighborhood. This model retains the underlying lattice of points of a conventional Potts model but delocalizes and smoothes point interactions, demonstrating significant improvement in terms of improved accuracy and reduced lattice bias. The tradeoff is additional computational complexity and a substantially larger neighborhood size $[37,38]$.

Another solution is to evolve the system on a random lattice, which by definition lacks the long-range order and symmetry that give rise to lattice effects. Such an approach was applied by Jannsens [13] in a cellular automaton model. To demonstrate this concept, we computed boundary energy as in Figure 1 for a three-dimensional random lattice. A $32 \times 32 \times 32$ system is filled with points at random positions using a density of 8 points per unit cell. Pairs of points separated by a distance of 1 or less are considered to be neighbors. The points are again assigned to one of two grains, and the boundary inclination is varied. The interfacial energy is considered to be the number of unlike neighbors at a point, normalized by the total number of neighbors. The same instantiation of random points is used for all calculations. As shown in Figure 2a, because points have different local environments, a distribution of energy values remains, but the systematic variation of boundary energy with boundary inclination has been erased.

The standard deviation in boundary energy for a 30x30x30 system is about $1.4 \%$ of the mean. However, the spread in boundary energies increases to over $10 \%$ of the mean as the volume sampled decreases. Thus, although boundary energy is isotropic with respect to boundary inclination, it may vary substantially due to local point configurations, especially when the number of points sampled is small. In the Janssens model [13], this source of error is noted, but it does not affect grain growth since the boundary motion rule in the cellular automaton depends only on local geometry and not on the grain boundary energy. (A corollary disadvantage is that the cellular automaton model cannot incorporate any variations in grain boundary energy, including physically based ones.) Changing the cellular automaton to treat grain boundary energy as a thermodynamic quantity would essentially generate a Potts model, and the Potts model for grain growth on a static irregular grid exhibits severe lattice pinning. Basically, if a boundary 
encounters a location with a high local energy anisotropy (typically associated with a low local point density), the boundary may become pinned in a low energy configuration in which any attempted state change increases the system energy. Boosting the temperature in an attempt to mitigate these effects resulted in generally disordered boundaries before the pinning effect was removed.

Figure $2 \mathrm{~b}$ shows that the random lattice also eliminates the systematic anisotropy in effective mobility, but that a distribution of mobilities remains due to local heterogeneities in the arrangement of points. It is interesting to observe that a particular point arrangement can affect the mobility of any boundary that includes it; thus, Figure $2 \mathrm{~b}$ contains lines of high mobility boundary inclinations that all contain an unusual local point configuration. These lines depend upon the details of the point distribution, so would appear in different places in different random lattice instantiations.

a)

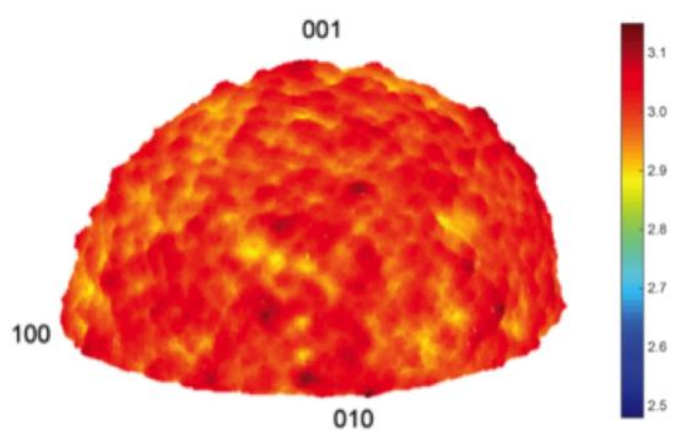

b)

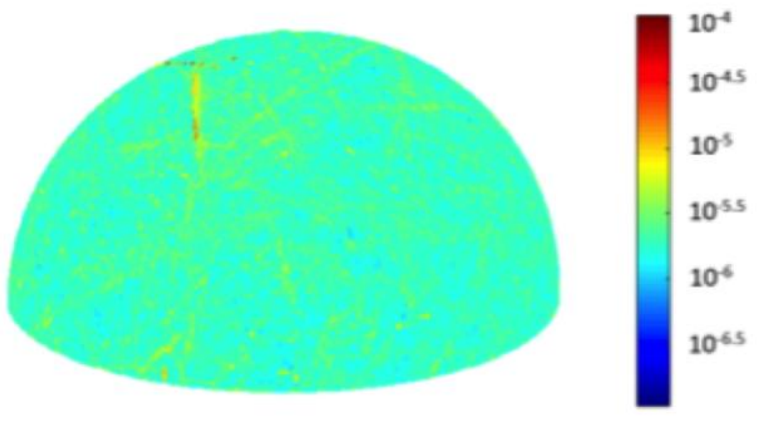

Figure 2: (a) The normalized boundary energy and (b) the effective boundary mobility as a function of boundary inclination of a static random lattice. There is no systematic correlation between boundary inclination and boundary properties, but some statistical variation is observed and is due to local variations in point neighborhoods.

In this paper, we derive a discrete, thermodynamic model for microstructural evolution that operates on a time-evolving set of random points. The randomness of the points prevents a systematic lattice bias, and the time evolution eliminates persistent inhomogeneous regions that interfere with boundary motion. The resulting method preserves some of the computational efficiency of the Potts model, but removes the lattice artifacts that limit its applicability. 


\subsection{The Material Point Monte Carlo (MPMC) method}

The MPMC model operates by creating and eliminating randomly distributed simulation points that store local materials data (grain orientation, local stress/strain, recrystallization state, etc.). At the onset of a simulation, a system volume is created, a point density per unit volume is chosen, and $N$ material points are placed at random in the volume. Each point is assigned materials data based on the desired initial microstructure. During each time step, $N$ points are selected at random, each of which is moved to a new random position where it takes on materials data based on its new neighborhood. Since the original materials data is discarded upon relocation, the algorithm essentially involves creation and destruction of material points. This sequence of steps is illustrated in Figure 3.

In grid-based systems, neighborhoods are assigned based on proximity. For example, most 3D Potts model simulations calculate interactions within the first three neighbor shells of a site on a simple cubic lattice. In the MPMC method, points are considered to be neighbors if they are closer than some cutoff distance from one another. In other words, a point will only be influenced by other points that fall within some sphere centered about itself, as shown in Figure 4(a).

When a new point is introduced at a location, it has no history, and needs to have its material data assigned in a physically meaningful way. Here, a statistical mechanics approach is used, which allows us to predict the probability of any state given the energy distribution of states (i.e. the partition function). Specifically, the probability of a state of energy $E_{i}$ is simply:

$$
p_{i}=\frac{e^{-\frac{E_{i}}{k T}}}{Z}
$$

where $\mathrm{Z}$ is the local partition function term, defined as the sum of the Boltzmann factors of the $q$ possible energy states: 


$$
Z=\sum_{j=1}^{q} e^{-\frac{E_{i}}{k T}}
$$

As an example, consider the case of grain growth, where the energy term is similar to that used in the Potts model, such that:

$$
E_{i}=\eta_{i}+\frac{1}{n_{s}} \sum_{j=1, j \neq i}^{n_{s}} \gamma_{i j}
$$

Here, the energy of material point $i$ is the sum of its bulk energy $\eta_{i}$ and the interfacial energy between $i$ and each of its $n_{s}$ neighbor points, where $\gamma_{i j}$ is the interfacial energy factor between points $i$ and $j$. (When $i$ and $j$ belong to the same grain $\gamma_{i j}=0$.) Note that the interfacial energy is normalized by the number of points in the neighborhood, so that points with different $n_{s}$ but identical grain identity ratios have the same absolute energy values. This is especially important for modeling systems with both interfacial and volumetric energy terms.

According to equation (5) high energy states (e.g. material points with no like neighbor points) are extremely improbable. Therefore, newly created material points are only allowed to adopt spins already held by at least one neighboring site. This does not change, in the energy-temperature regime used, the behavior of the system, but does tremendously improve computational performance of the method. One important distinction of this method from other off-lattice Monte Carlo methods [13], is that all point destruction-creation steps are accepted, and the thermodynamic behavior of the system is captured in the state selection process. 


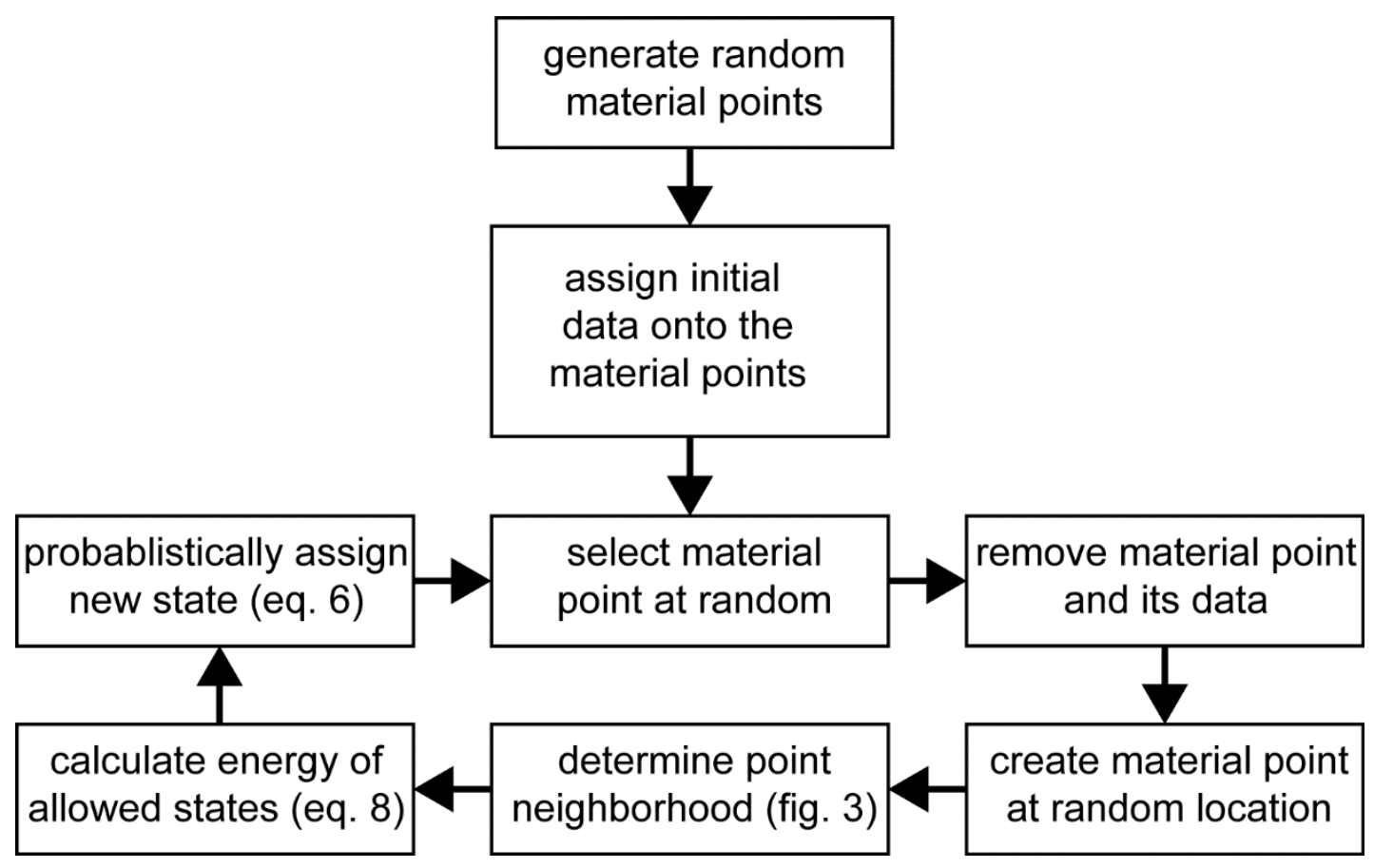

Figure 3: A flow chart for the Material Point Monte Carlo algorithm. 


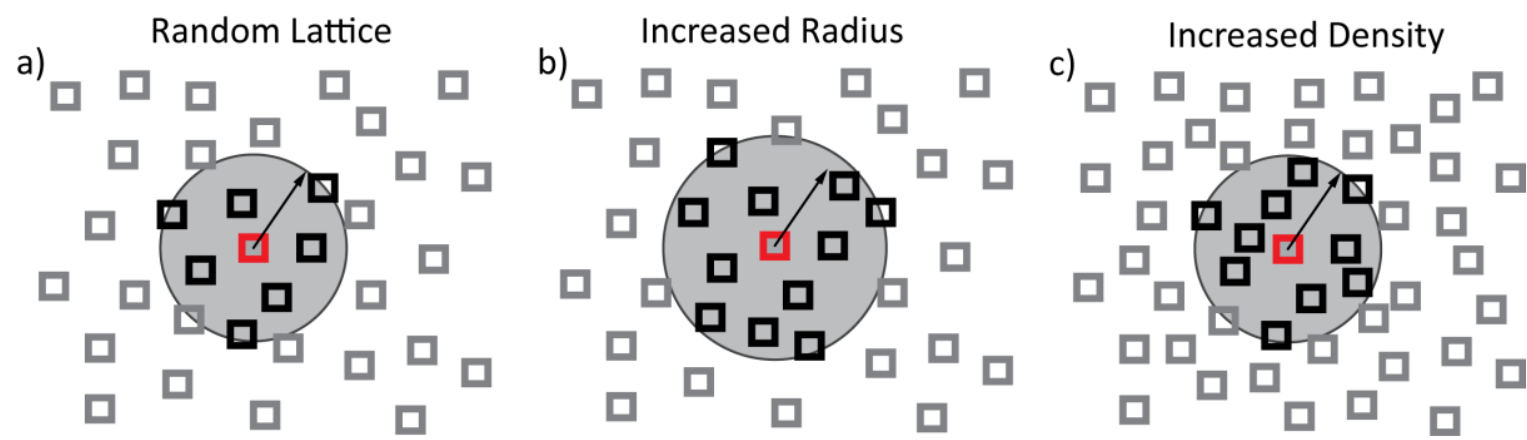

Figure 4: An illustration of the parameters in the MPMC. (a)The random points that fall within a radius are considered part of a given point's neighborhood. Both (b) increasing the neighborhood radius or (c) increasing the density of points per area can be used to increase average number of nearest neighbors, but the overall outcomes are distinct.

As shown in Figure 4, there are two ways to increase the average number of neighbors per material point and approach the limit of a continuous medium. Increasing the material point density is equivalent to increasing the system size $N$. While it improves accuracy and decreases error, it also multiplies the computational cost per time step, which scales as $N$ (as discussed in section 3.4). Increasing the neighborhood radius at constant system size effectively decreases the resolution. In practice, for a given system size, the neighborhood radius is chosen to achieve the desired average number of neighbors. In the following examples, the neighborhood radius and point density are selected to yield approximately 33.5 neighbors per material point. This value is chosen because it is on the same order as the 26 nearest neighbors used in most three-dimensional Potts model simulations; it minimizes the frequency of points with no neighbors [13]; and it gives good results in a reasonable computational timeframe. Other average neighbor counts can also be used successfully, depending on the resolution desired.

\section{Results}

\subsection{Validation and Verification: Isolated grains}

The goal of the MPMC is to simulate microstructural evolution driven by interfacial energy minimization (i.e. curvature-driven motion) and by bulk energy minimization (e.g. strain-assisted boundary migration). To verify that both driving forces are properly reproduced, we verify the MPMC for the simplest case of an isolated grain in a matrix. 


\subsubsection{Curvature-driven motion}

In order to confirm that the MPMC approach gives rise to curvature-driven motion, we consider a circular grain embedded in a matrix grain at a simulation temperature of zero, as shown in Figure 5(a). In order to reduce the total interfacial free energy, such a grain should shrink such that grain area decreases linearly with time, i.e. $d A / d t=$ constant $[39$, 40].

When a new material point is introduced at $T=0$, it will adopt the grain identity of the majority of its neighbors. Thus, the system is divided into three regions, as shown in Figure 5(a): the stable embedded grain, where any new point will adopt the embedded grain identity; the matrix region, where any new point will adopt the matrix grain identity; and a mobile region inside the embedded grain, where new points will on average adopt the matrix grain identity and move the grain boundary inward.

Because every material point is updated once (on average) per Monte Carlo timestep, the change in area per unit time should be equal to the area of the mobile region:

$$
\frac{d A}{d t}=A_{m}
$$

where the mobile area $A_{m}=\pi \Delta R^{2}$. The boundary of the stable region [dashed line in Figure 5(a)] is the location where the neighborhood is half-filled by the embedded grain, i.e. the embedded grain area $A_{e}=\pi r^{2} / 2$. The area of overlap $A_{o}$ between two circles with centers $R-\Delta R$ apart is given by

$$
A_{o}=r^{2} \cos ^{-1}\left(\frac{\Delta R^{2}-2 R \Delta R+r^{2}}{2 R^{2}-2 R \Delta R}\right)+R^{2} \cos ^{-1}\left(1+\frac{\Delta R^{2}-r^{2}}{2 R^{2}-2 R \Delta R}\right)-\frac{1}{2}\left[\left(r^{2}-\Delta R^{2}\right)\left((2 R-\Delta R)^{2}-r^{2}\right)\right]^{1 / 2}
$$

Setting $A_{o}$ equal to $\pi r^{2} / 2$, we can solve (numerically) to find $\Delta R$ as a function of the scaled embedded grain radius, $R / r$. We then compute the mobile area as a function of $R / r$ and by equation (8) find that $d A / d t$ is constant for $R>r$, as shown in Figure 5(b), 
confirming that the embedded grain evolves with the correct kinetics for curvature-driven growth.

a)

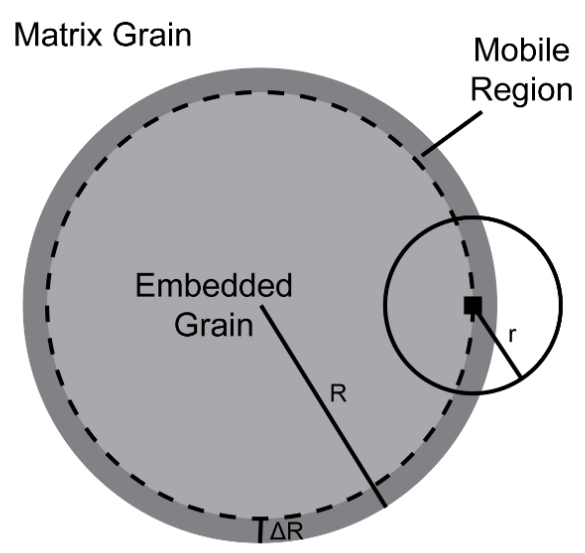

b)

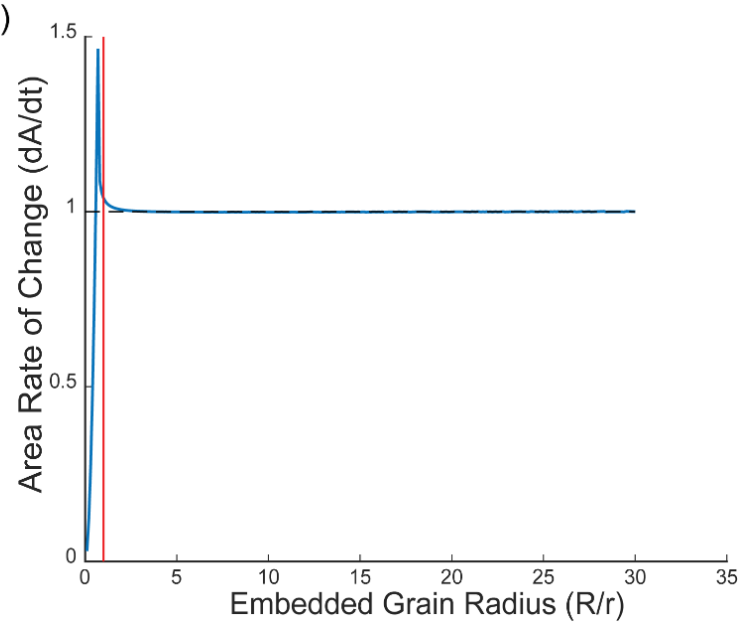

Figure 5: (a) A schematic of a circular grain of radius $R$ embedded in a matrix grain, with a dashed line representing the demarcation between new points selecting the embedded grain or the matrix grain. (b) The change in area per unit time of the embedded grain as a function of its radius $R$ relative to the neighborhood radius $r$. Since $d A / d t$ is constant for $R>r$, the grain evolves by curvature driven growth.

To verify the operation of the MPMC code, the case of a spherical grain embedded in a matrix grain is tested. As shown in Figure 6, the spherical grain shrinks such that the grain radius is proportional to the square root of time, which is consistent with curvaturedriven interface motion 

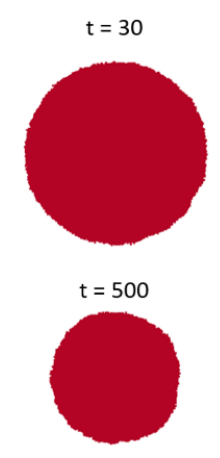
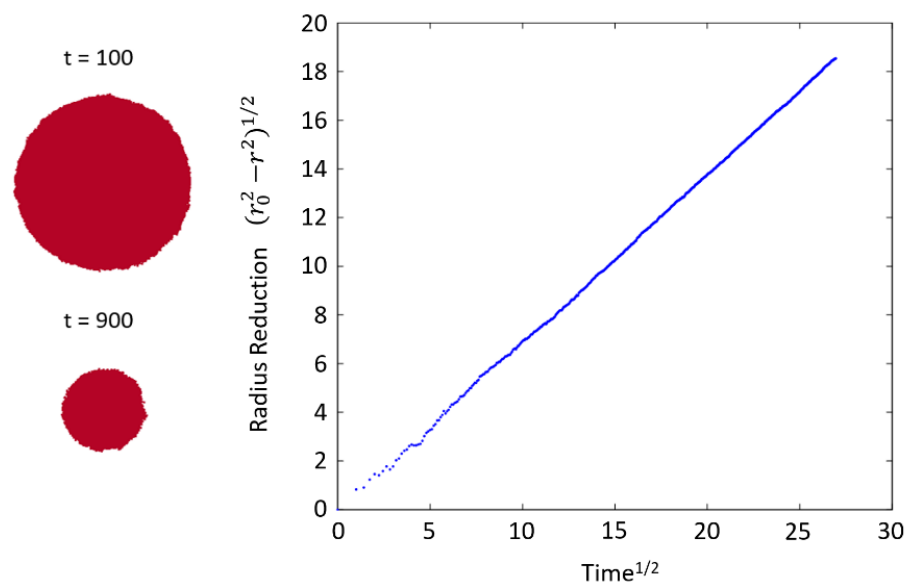

Figure 6: Left: MPMC time snapshots of a spherical grain embedded in a matrix grain, shrinking to minimize interfacial energy. Right: A graph demonstrating that the spherical grain shrinks with the parabolic kinetics expected for curvature-driven evolution.

\subsubsection{Bulk free energy driving forces}

In order to verify that the MPMC correctly captures bulk free energy driving forces, we consider a bicrystal with a flat grain boundary where one grain has a strain energy density $\eta=0$, and the other has a finite strain energy density. In principle, the boundary velocity, $v$, should be proportional to the difference in strain energy density, $\Delta \eta$. However, in the Potts model, this scaling is not observed, as shown in Figure 7(a). Specifically, at low temperature the boundary does not move below a threshold strain energy density, since nucleation of surface steps is energetically prohibited for $\Delta \eta$ below a lattice-dependant value. Even at relatively high simulation temperatures, the effects of the nucleation barrier result in a nonlinear relationship between $v$ and $\Delta \eta$. In contrast, the MPMC reproduces the expected scaling over several orders of magnitude in strain energy density, as shown in Figure 7(b). The limits of the scaling regime occur above $\Delta \eta=0.4$, where the driving force is sufficient to kinetically roughen the boundary, and below the case of $\Delta \eta=0.003$, where it becomes difficult to distinguish driven motion from random fluctuations. 
a)

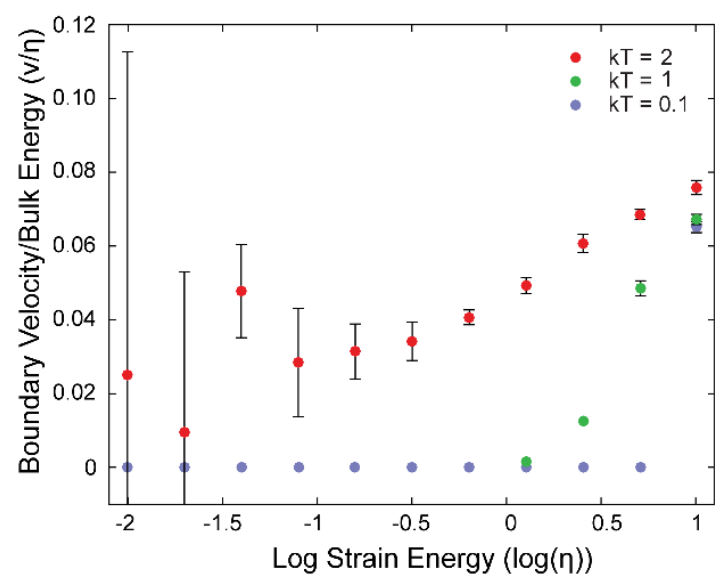

b)

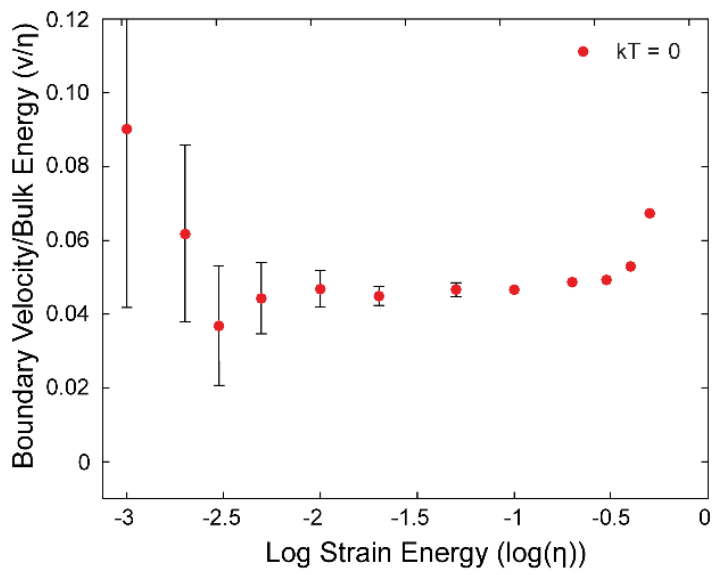

Figure 7: A comparison of how bulk energy in (a) the Potts model and (b) MPMC can drive a flat boundary in a bicrystal along the [100] plane in simulation frame. Ideally, the plot should form a flat line until strain energy becomes small enough that random fluctuations dominate. The MPMC is much closer to this ideal, due to a lack of lattice artifacts that oppose boundary motion.

\subsection{Lattice Artifacts in isotropic grain growth}

The primary motivation for developing the MPMC method is to minimize lattice artifacts in microstructural evolution simulations. Because isotropic grain growth is quite sensitive to lattice effects, we perform a 3D MPMC simulation starting with one million unique material points placed at random; material point energy is given by equation (7) with bulk energy $\eta_{i}=0$ and $k T=0$. As expected in isotropic grain growth, grains grow as compact shapes that maintain 120 degree angles at triple lines (Figure 8), and after a brief transient period while grains were under the neighborhood size, the average grain radius increases proportional to time with an exponent of 0.5 (Figure 9).

To test for simulation lattice artifacts, the grain boundary plane distribution in sample space is computed for the MPMC microstructures and compared with equivalent microstructures generated by Potts model simulation with $k T=0.1$. The method and code for the grain boundary plane distribution calculation was developed by Lieberman et al. [41]. Note that the code for the grain boundary normal calculations requires a binning of the material points onto a coarser set of points in a cubic grid. 
As shown in Figure 10, the Potts model evinces strong peaks at low-index grain boundary plane normal directions, particularly [001], which is expected, given the energetic landscape of the neighborhood in cubic systems. This contrasts the grain boundary plane distribution of the MPMC, which is much more uniform without any systematic or significant peaks. This supports the assertion that the MPMC microstructural evolution does not share the lattice artifacts found in the conventional Potts model.

For the Gaussian kernel Potts model, Mason et al. also examined the distribution of grain boundary normals to determine the extent of lattice artifacts [37]. For two-dimensional grain growth, they find that the grain boundary normal distribution approaches that of an ideally isotropic polycrystal as the kernel size increases, thus minimizing the effect of the underlying hexagonal lattice symmetry. A key difference is that in the Gaussian kernel method, lattice artifacts diminish (but do not disappear) as the kernel is optimized, while in the MPMC, the dynamic random lattice has no persistent artifacts regardless of interaction radius. 

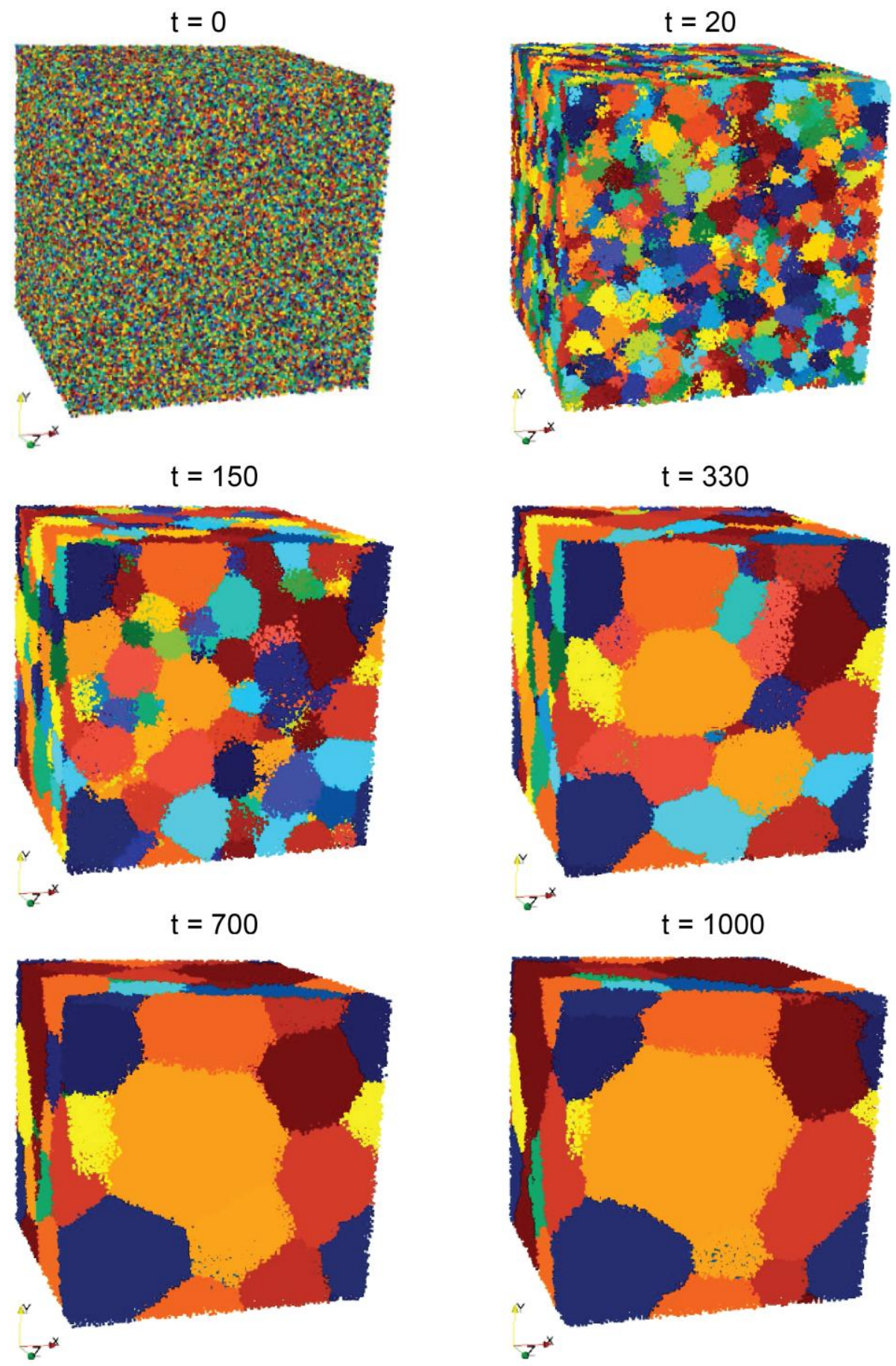

Figure 8: Time evolution of isotropic polycrystalline microstructures in the MPMC approach. The grains grow in a manner consistent with that of ideal grain growth. Note that the visualization of the random points as dots allows some vision into the interior of the micrograph. 


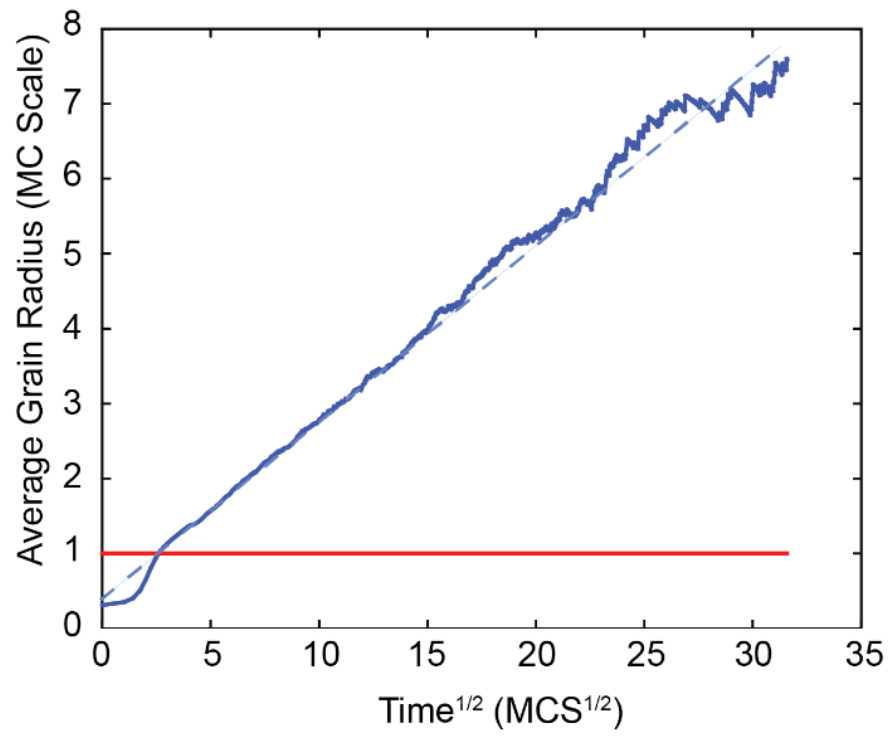

Figure 9: Grain growth kinetics in the MPMC starting from single-site grains. Once the average grain radius exceeds the material point neighborhood radius (red line), the correct parabolic time-radius relationship for normal grain growth (dashed line) is obtained.
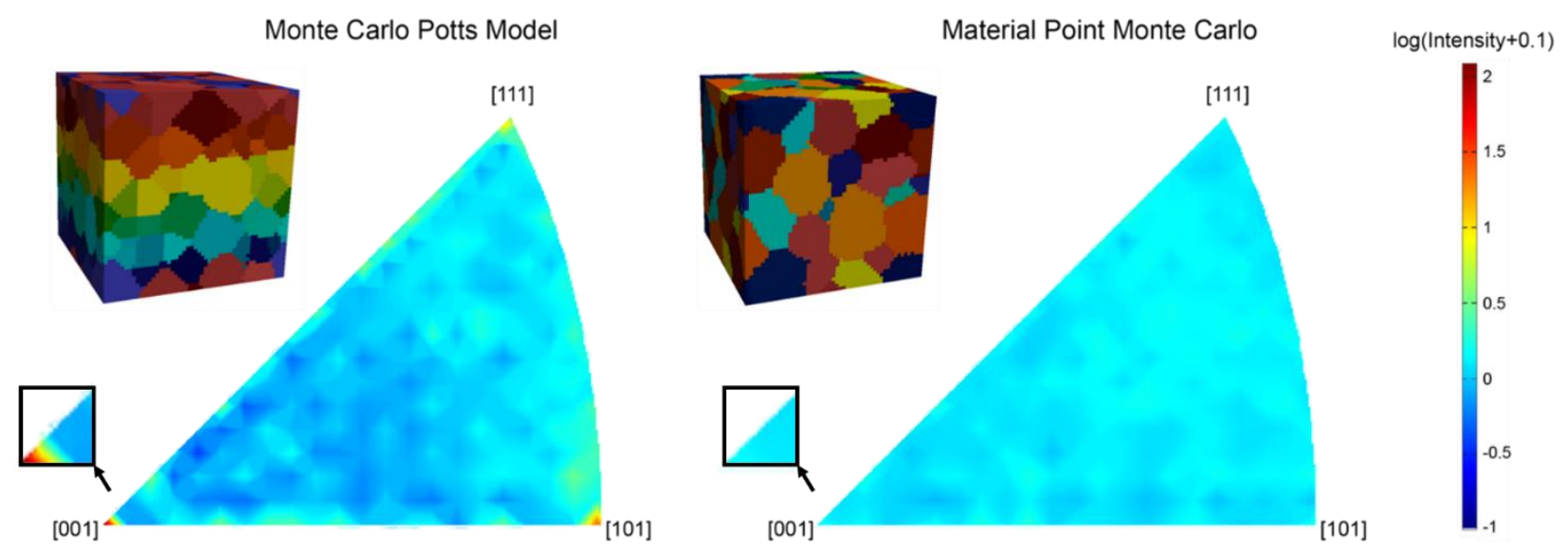

Figure 10: Grain boundary inclination distributions of the conventional Potts model (left) and the MPMC (right), Note that the peaks at [001], [101], and [111] are very sharp in the Potts microstructure, and that the scale used is logarithmic. The upper insets are visual representations of the microstructures used; the lower insets highlight the boundary plane normal distributions near [001]. Note that the MPMC microstructure here was binned into a coarser cubic structure for the grain boundary normal calculations. 


\subsection{Grain stagnation during lattice deformation}

One key advantage of using a random arrangement of points is that it remains a random arrangement of points after affine transformations[13]. This is particularly helpful when attempting to deform a system. The regular lattice used in the Potts model is not amenable to deformation. When the Potts lattice is "stretched," the energetic interactions between neighboring sites become more anisotropic. An example of a three-dimensional Potts model with no strain and with a modest $10 \%$ strain is shown below, using a $256^{3}$ grid size and a simulation temperature of $k T=0.1$. (Grains were first grown normally for 125 MCS to prepare the initial microstructures.) With even $10 \%$ change in interaction energy between directions, most grains rapidly become pinned. A few rare events result in some grains overcoming this pinning and growing abnormally. This abnormal grain growth does not have a physical basis, and this behavior is entirely the result of lattice pinning effects.
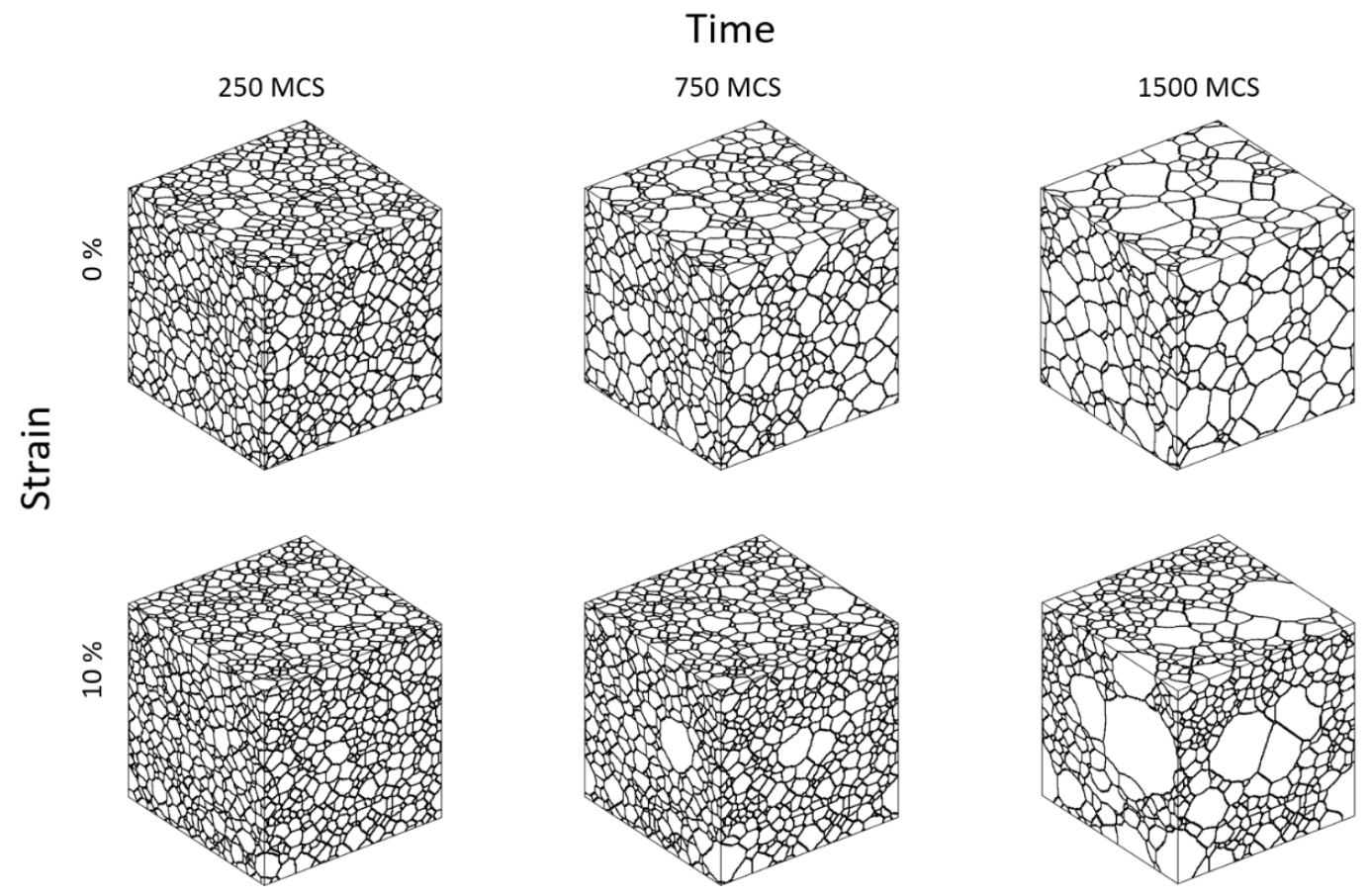

Figure 11: Grain growth in a Potts model in which the underlying lattice has been strained $10 \%$ in the $y$ direction, resulting in unphysical grain shapes, growth stagnation, and abnormal growth events. 
In contrast, the MPMC simulation volume can be deformed with no negative effect, since during volume conserved deformation the average number of nearest neighbors remains constant and the spatial distribution of neighbors remains random. An example of a constant-volume system deformation is shown in Figure 12, where the system undergoes uniaxial tension for 290 time steps under a strain rate of 0.005 per MC time step with a Poisson ratio of 0.3 . Note that grain shapes are governed by the competition between geometric deformation and interfacial energy minimization. Grain stagnation does not occur, even at very high strains.

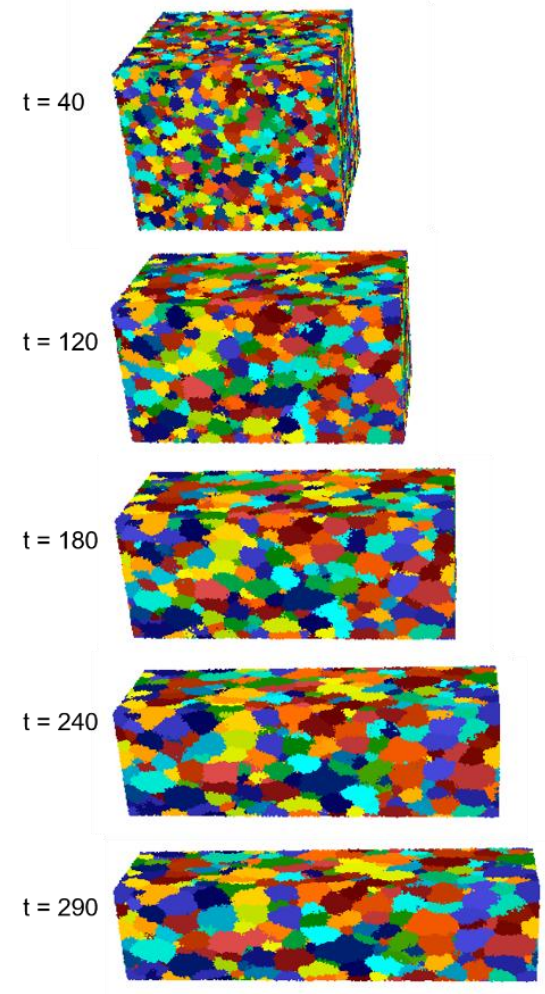

Figure 12: MPMC grain growth in a system that is continuously deformed in tension to a strain of 2.4 at strain rate 0.005 per time step with a Poisson ratio of 0.3 . The geometric elongation of grains competes against interfacial energy minimization, and grains grow continuously.

\subsection{Computational considerations}

A major reason that the Potts model continues to be used to simulate microstructural evolution is its exceptional computational efficiency relative to other methods such as 
phase field and front tracking. In its simplest form, the Potts model requires on order $N$ calculations per Monte Carlo timestep, and significant computational speedups are possible[42]; the Potts model also parallelizes efficiently via spatial decomposition[43].

Like the Potts model, the MPMC method also requires on order $N$ calculations per Monte Carlo timestep if spatial binning is used to assist in neighbor identification, and it should parallelize well. However, the necessity for real number operations increases the computational overhead compared to the Potts model.

In order to estimate the additional cost of the MPMC method, we compared the MPMC with SPPARKS[43], a standard Potts model code, on a model 3D grain growth system. The initial condition consists of $10^{6}$ points with unique identities; the system coarsens under isotropic grain growth at a simulation temperature $k T=1.0$ until 100 grains remain. Both codes were run on a single processor of a 2011 MacBook Pro. Two version of the SPPARKS simulation were performed: one representing the simplest order- $N$ implementation of the Potts model (rejection kMC, random sweeps, no masking), and the other using the most efficient speedup (exact kMC), both of which are described in the SPPARKS documentation online[44]. The MPMC code required 300 minutes to complete the simulation, compared with the order- $N$ Potts at 100 minutes and the optimized Potts at 20 minutes. Although it is difficult to compare code performance directly, the results indicate that the current version of the MPMC model is comparable to the analogous Potts implementation in terms of computational efficiency. Furthermore, many of the speedup strategies available for the Potts model should be applicable to the MPMC method as well, suggesting that an order of magnitude increase in performance is realistic to expect.

There is not currently a standard, open source, three-dimensional version of the Gaussian kernel based Potts model code developed by Mason et al. [37]. In comparisons with our own version of the kernel based Potts code, the MPMC was substantially faster, due to the much smaller interaction radius between points. However, only a very basic implementation of the kernel based method was used, and speedups that have been made to the method [38] were not employed. It is likely that both approaches are capable of 
significantly improved computational efficiency, so that which will be faster is not yet obvious - and may even vary with application.

While direct testing against the static random lattice cellular automaton model of Janssens [13] has not been carried out, it is expected that the MPMC would be notably slower. The primary trade-off for replacing the static random lattice with an evolving random grid is that neighborhoods must be continually recalculated. On the other hand, the thermodynamic and kinetic bases of the MPMC enable more physically realistic systems.

\section{Conclusions}

While the Monte Carlo Potts model is widely used to simulate microstructural evolution, it suffers from significant artifacts of the underlying simulation lattice, including substantial anisotropy in interfacial energy and mobility. In response, we have developed the Material Point Monte Carlo method, which operates on a spatially random set of discrete material points. Using a statistical mechanics scheme, points are randomly relocated and their material data are updated at each timestep. Because material points are randomly distributed, and remain so under affine deformations, the MPMC eliminates artifacts of the simulation grid that occur in lattice-based methods such as the Monte Carlo Potts model. Using single interface model systems, the MPMC is verified to correctly reproduce grain boundary motion caused by bulk and interfacial driving forces. When applied to simulate normal grain growth, the MPMC reproduces the correct kinetics and avoids the preferential grain boundary orientations seen in lattice-based models. When simulating grain growth during deformation, the MPMC is able to capture simultaneous grain growth and plastic elongation in a manner that lattice-based models cannot.

\section{Acknowledgements}

This work was performed at Carnegie Mellon University and was supported by the United States National Science Foundation award DMR-1307138. P.E.G. would also like to acknowledge the support of the John and Claire Bertucci Graduate Fellowship 
Foundation. The authors would also like to thank Brian DeCost for his assistance with the Potts model deformation test.

\section{References}

[1] R.B. Potts, Mathematical Proceedings of the Cambridge Philosophical Society, 48 (1952) 106-109.

[2] P.S. Sahni, G.S. Grest, M.P. Anderson, D.J. Srolovitz, Physical review letters, 50 (1983) 263.

[3] M.P. Anderson, D.J. Srolovitz, G.S. Grest, P.S. Sahni, Acta Metallurgica, 32 (1984) 783-791.

[4] D.J. Srolovitz, M.P. Anderson, P.S. Sahni, G.S. Grest, Acta Metallurgica, 32 (1984) 793-802.

[5] E.A. Holm, M.A. Miodownik, A.D. Rollett, Acta Materialia, 51 (2003) 2701-2716.

[6] E.A. Holm, G.N. Hassold, M.A. Miodownik, Acta Materialia, 49 (2001) 2981-2991.

[7] M. Kobayashi, Y. Takayama, H. Kato, MATERIALS TRANSACTIONS, 42 (2001) 2307-2315.

[8] I. Steinbach, F. Pezzolla, B. Nestler, M. Seeßelberg, R. Prieler, G.J. Schmitz, J.L.L. Rezende, Physica D: Nonlinear Phenomena, 94 (1996) 135-147.

[9] S.G. Kim, D.I. Kim, W.T. Kim, Y.B. Park, Physical Review E, 74 (2006) 061605.

[10] I. Steinbach, F. Pezzolla, Physica D: Nonlinear Phenomena, 134 (1999) 385-393.

[11] C.E. Krill Iii, L.Q. Chen, Acta Materialia, 50 (2002) 3059-3075.

[12] D. Raabe, Annual Review of Materials Research, 32 (2002) 53-76.

[13] K.G.F. Janssens, Modelling and Simulation in Materials Science and Engineering, $11(2003) 157$.

[14] J. Geiger, A. Roósz, P. Barkóczy, Acta Materialia, 49 (2001) 623-629.

[15] Y. Liu, T. Baudin, R. Penelle, Scripta Materialia, 34 (1996) 1679-1683.

[16] R. Carel, Department Materials Science and Engineering, Massachusetts Institute of Technology, 1995, pp. 266.

[17] W. Fayad, C.V. Thompson, H.J. Frost, Scripta Materialia, 40 (1999) 1199-1204. [18] H.J. Frost, C.V. Thompson, C.L. Howe, J. Whang, Scripta Metallurgica, 22 (1988) 65-70.

[19] K. Kawasaki, T. Nagai, K. Nakashima, Philosophical Magazine Part B, 60 (1989) 399-421.

[20] L.A. Barrales Mora, Mathematics and Computers in Simulation, 80 (2010) 14111427.

[21] D. Moldovan, D. Wolf, S.R. Phillpot, A.J. Haslam, Acta Materialia, 50 (2002) 33973414.

[22] S.P.A. Gill, A.C.F. Cocks, Acta Materialia, 44 (1996) 4777-4789.

[23] M. Elsey, S. Esedog- lu, P. Smereka, Journal of Computational Physics, 228 (2009) 8015-8033.

[24] A. Kuprat, D. George, G. Straub, M.C. Demirel, Computational Materials Science, 28 (2003) 199-208.

[25] Z.T. Trautt, Y. Mishin, Acta Materialia, 60 (2012) 2407-2424.

[26] E.A. Holm, J.A. Glazier, D.J. Srolovitz, G.S. Grest, Physical Review A, 43 (1991) 2662. 
[27] E. Holm, C. Battaile, JOM, 53 (2001) 20-23.

[28] E. Ising, Zeitschrift für Physik, 31 253-258.

[29] A.D. Rollett, Progress in Materials Science, 42 (1997) 79-99.

[30] N. Metropolis, A.W. Rosenbluth, M.N. Rosenbluth, A.H. Teller, E. Teller, The Journal of Chemical Physics, 21 (1953) 1087-1092.

[31] O.M. Ivasishin, S.V. Shevchenko, N.L. Vasiliev, S.L. Semiatin, Materials Science and Engineering: A, 433 (2006) 216-232.

[32] A.D. Rollett, D. Raabe, Computational Materials Science, 21 (2001) 69-78.

[33] E.A. Holm, J.A. Glazier, D.J. Srolovitz, G.S. Grest, Physical Review A, 43 (1991) 2662-2668.

[34] L. Mittag, M.J. Stephen, Journal of Physics A: Mathematical, Nuclear and General, 7 (1974) L109.

[35] G.S. Grest, M.P. Anderson, D.J. Srolovitz, Physical Review B, 38 (1988) 47524760.

[36] J.A. Glazier, M.P. Anderson, G.S. Grest, Philosophical Magazine Part B, 62 (1990) 615-645.

[37] J.K. Mason, J. Lind, S.F. Li, B.W. Reed, M. Kumar, Acta Materialia, 82 (2015) 155166.

[38] J.K. Mason, Acta Materialia, 94 (2015) 162-171.

[39] M. Hillert, Acta Metallurgica, 13 (1965) 227-238.

[40] J.E. Burke, Transactions of the American Institute of Mining and Metallurgical Engineers, 180 (1949) 73-91.

[41] E.J. Lieberman, A.D. Rollett, R.A. Lebensohn, E.M. Kober, Modelling and

Simulation in Materials Science and Engineering, 23 (2015) 035005.

[42] G.N. Hassold, E.A. Holm, Computers in Physics, 7 (1993) 97-107.

[43] S.A. Wright, S.J. Plimpton, P.T. Swiler, R.M. Fye, M.F. Young, E.A. Holm, Sandia Report, SAND97-1925 (1997) 1-44.

[44] SPPARKS Documentation, Sandia National Lab, http://spparks.sandia.gov/doc/solve_style.html, (2016). 